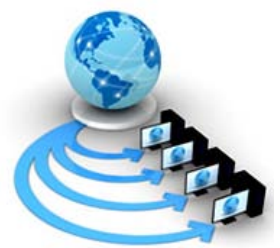

Volume 9, No. 2, March-April 2018

International Journal of Advanced Research in Computer Science

REVIEW ARTICLE

\author{
Available Online at www.ijarcs.info
}

\title{
THE POWER OF CLOUD COMPUTING: INTEGRATION AND INTELLIGENCE
}

\author{
D.Venkatesh \\ Department of Information Technology \\ Chaitanya Bharathi Institute of Technology \\ Hyderabad, Telangana, India
}

\author{
Suresh Pabboju \\ Department of Information Technology \\ Chaitanya Bharathi Institute of Technology \\ Hyderabad, Telangana, India
}

\author{
Vijay Kumar Damera \\ Department of Information Technology \\ Mahatma Gandhi Institute of Technology \\ Hyderabad, Telangana, India
}

\begin{abstract}
This paper is based on the development of cloud computing to propose a new scenario for enterprise information systems. From a view of the cloud as an infrastructure technology, it is possible to think of inter-organization integrations in a more efficient way, in a great possibility of data mining with the data of different companies and in its later analysis of data for better decision making. For non-competitive environments, the scenario of integration and intelligence defended is extremely probable and there is already movement in this direction. However, it is understood that data privacy and copyright issues still require further study, in addition to market dominance by few players with unknown consequences.
\end{abstract}

Keywords: Cloud Computing; Data Mining; Business Intelligence Component;

\section{INTRODUCTION}

As is often the case with infrastructure technologies, today there is a process of standardization and commoditization of IT applied to enterprise information systems and, in particular, to integrated management systems. It is not uncommon to face the challenge of choosing between different solutions and it becomes very difficult to find differentials among suppliers.

Cloud computing can be translated as a new step in this direction of standardization: IT as a utility. With cloud computing, we have a number of advantages such as scalability, increased collaboration, increased reliability and freedom for the company to focus on its core business. This paper proposes to discuss the integration made possible by this platform, one of the main benefits created, and especially its possible consequences. Such integration is achieved precisely by the possibility of becoming common to the infrastructure of several different companies (including different sectors), which ends up providing an unequalled amount of data. With the possibility of crossing this data, we would face predictability and a foundation for unimaginable business intelligence. The paper will navigate this initial outline of the scenario, trying to analyze what could be done, using examples such as ERPs (Enterprise Resource Planning) in the cloud, and what are the main challenges for this to become a reality.

\section{A. Formulation of the Problem Situation}

With the contextualization presented during the introduction, the problem situation in question can be indicated by the following question: With a mass adoption of companies by cloud management systems, what could be done in terms of integration and intelligence, through the available data?. Responding to this questioning will be the focus of this study.

\section{B. Objectives}

The objectives of the study are to analyze the recent history and future of enterprise information systems, especially addressing the topic of cloud computing, and propose a possible scenario of integration and business intelligence through a mass adoption of companies by such systems.

\section{Method}

The research method used in this work is characterized by a bibliographical research, developed through a literature review on the subject. The bibliographic research allowed us to explore the theme in question, through consultation in scientific articles, books, magazines and websites, and is justified by the contribution to the development of the proposed scenario.

\section{THE FUTURE OF IT}

Nicholas Carr in the book "Is IT Everything?" Supports a provocative and controversial argument about IT. He quotes the following line from Dell's founder Dell Dell: "In the long run, every technology tends to have low-cost standards" [1]. For Carr, TI "is perhaps best understood as the latest in a series of widely adopted technologies that have reshaped the productive sector over the past two centuries" [2]. So it was with the electric power, with the railroads and the telecommunications networks. "All these technologies have offered opportunities for the most agile and visionary companies to take advantage of the competition." However, "when they became widespread everywhere they became inputs" [2].

However, this generalization is not valid for all IT. It is necessary to divide the use of IT as solely support to the business and as a way to enable the creation of new values and business models. It is important to recognize what IT hides: the knowledge and business processes of a company. "By adopting an ERP, the basic goal is not to put the software into 
production, but to improve business processes using information technology" [3]. IT can not be underestimated, but it must be rethought.

There are those who say IT will never stop evolving, that technological progress tends to infinity. This may even be true, but IT may run into hyper-efficiency, that is, "the technological process by which the performance of a technological product exceeds the requirements of the majority of its users" [4]. We can safely assume that all employees of a company will never use all the features of their office suite. The standardization by hyper efficiency has already reached the softwares. "In 1998, Ray Lane, then president of Oracle, said that 'customers do not see even 5\% of the differences in SAP, PeopleSoft and us"' [5].

It is worth mentioning that the correlation between a company's profitability and IT investments is a debatable point and usually complicated to prove. Carr cites a number of studies in his book, such as Brynjolfsson and Hitt, 1996. They concluded that IT increases corporate productivity. However, when analyzing the financial performance of 370 large US companies, they concluded that the data presented "little evidence of an impact of IT on above-normal profitability" [6]. In 1997, Prasad and Harker analyzed 47 leading US retail banks and "found no evidence that IT capital spending had increased the company's profitability" [6].

\section{Cloud Computing}

Today, IT gives signs that the process of commoditization and standardization has reached another level: cloud computing. This new concept frees companies from the complicated bindings of hardware and software that can "lease" them, outsource IT to the extreme, transform it into utility - like electricity and telephony.

Cloud computing is a concept that describes "a computing environment based on a massive network of servers, whether virtual or physical" [7]. Therefore, grid computing, parallel processing, autonomic computing, and virtualization are part of this process. "A simple definition can then be a set of resources such as processing capacity, storage, connectivity, platforms, applications and services available on the internet" [7].

Cloud computing follows the logic of everything-as-aservice and, conceptually, it can be separated into several layers, such as: Infrastructure as a Service; backup as a service (Backup as a Service); platform as a service (Plataform as a Service); database as a service (Database as a Service); and Software as a Service (Software as a Service). In "The Experience Economy" (1999), Pine and Gilmore refer to the progression of economic value as an evolution of economic offerings, starting with the extraction of commodities, passing on the production of goods, delivering services, providing experiences and, finally, the conduction of transformations. They maintain that each variation in the economic supply has a competitive differentiation compared to its competitors. A striking example is that today many manufacturers seek to differentiate their goods by offering larger guarantees, which are services. IT is following this trend. Today, for example, when you buy a few ASUS laptops, your customers gain one year of unlimited data storage space in the company's cloud, "ASUS Web Storage." In 2011, Google announced that it could offer laptop rentals for twenty dollars a month. Turning IT into service is also a strategic issue for business and cloud computing can have a big influence on this issue.

IaaS is basically having available computing resources ("virtual machines") over the network. It frees the IT department from the need to manage hardware, but there is still a lot of work with configuration and development. The US company Amazon was one of the first to offer Cloud services and is recognized as a major supplier of IaaS. It is worth mentioning how this offer came about. Amazon had large idle capacity in its data centers. What favored that the virtual store could risk in this new branch was, precisely, that in many moments the used infrastructure was around 10\%. It, like any company that is not in the cloud, needed to oversize its computing resources to meet demand spikes. The idea arose of renting this idle capacity so that third parties could enjoy it. Economics of scaling and scope are basic principles of cloud servers. According to Montella (2005)[8], we have economies of scale when cost decreases by increasing the quantity produced and economy of scope when the cost of producing two goods together is less than the cost of producing them separately.

At the other end, as opposed to IaaS, is the SaaS. In this layer, the company receives the software as a service and is the one that least needs interaction between a company's IT department and its service provider. Today there is a huge amount of SaaS layer applications on the Internet. Apps from Google Apps (Google Mail, Calendar, and Docs, for example), SalesForce.com and even AutoCAD WS. "Software-as-aService is a disruptive model. Your value proposition is the functionality offered and not the 'property' of the product. The basic idea is that you do not actually want a washing machine, but you want the laundry washed. SaaS offers that. You do not need to install a CRM [Customer relationship management] or ERP package, but you need the features "[9]. System updates, backup routines, and all the "sacrifices" that accompany "traditional" IT fall to SaaS, we just get what we need. The "new IT" is almost invisible, it's a cloud

Cloud computing allows companies to have a more elastic IT infrastructure, that is, it is used and contracted as needed in a pay-as-you-go model, avoiding over-or under-resizing of computing resources. The adoption of a solution of this style is also usually more agile. For companies up to medium-sized, cloud computing allows you to have a performance and IT sophistication that would be very costly to maintain. The economic advantage of cloud computing is mainly represented by the transformation of capex (capital expenditures) into opex (operating expenses), alleviating corporate cash flow, reducing IT infrastructure costs for companies, enabling IT resource allocation [9]. In this paper, we present the results of the study, which are presented in Table 1 . We can not fail to mention that cloud computing also provides the strength for business collaboration and allows for an investigation to work outside the company environment, ensuring greater flexibility, and decreasing deployment times in a truly drastic way, given the way delivery of their products via the internet.

On the other hand, there are still some challenges to overcome in the cloud in terms of ensuring security, privacy and cloud interoperability, not to mention international legal barriers. It should be noted, however, that a conscious choice of a cloud provider with well-defined service level agreements and SLAs can minimize such problems. Even if multiple systems run in parallel in the same environment, it is possible to segregate data and even network traffic, ensuring privacy and invisibility for the customer [10]. Making an analogy, companies already use outsourced companies to handle their net assets - banks. It is clear that many industrial secrets constitute the capitalist spirit of certain companies and should have an internal security policy, segregating what should or should not go to the cloud. It is still worth mentioning that one can resort to private clouds, or hybrid models with public clouds. All this is a process of maturing the industry. And, of course there is a share of natural resistance of companies to adhere to change. 


\section{ERPS IN THE CLOUD}

The SaaS model - Software as a Service - and also PaaS Platform as a Service - is gradually being increasingly adopted for enterprise information systems such as CRMs (Customer Relationship Management) and ERPs (Enterprise Resource Planning). The ease of deployment and approval, the ability to collaborate, and develop applications to attach to systems should be a good attraction. In order to conduct the paper, we will focus our approach on ERPs, mainly because of the amount of information it is able to store.

During the early 1990s, large companies began to implement ERPs - also known as Integrated Management Systems (GIS). By the end of the decade, the market was saturated and suppliers turned to small and medium-sized enterprises. In 2002, Mendes and Escrivo Filho elaborated a bibliographical review of several authors on the characteristics of an ERP, the main ones found were: ERP helps decision making; serves all areas of the company; has a single centralized database; allows greater control over the company; evolution of the MRPII; obtains information in real time; allows integration of business areas; has reference models; is a generic system; supports strategic planning; supports the need for information from the areas; supports the company's operations; is an organizational change tool; process orientation.

It is possible to realize that the ERP contains valuable information of a company and its database is rich for data mining, that is, the prospecting of data. In short, data mining consists of exploring a large volume of data in search of patterns and associations. Another related point is the practice of Business Intelligence.

There are already ERP initiatives in the cloud. Just search for keywords like "ERP Cloud Computing" in Internet search engines that come up with some answers, but for now they are still modest. "Traditional ERP systems still dominate the market widely. Currently, ERP solutions in the SaaS model account for only $2 \%$ of global ERP market revenue, according to Forrester Research. Still, Forrester expects worldwide cloudbased ERP spending to grow about 21 percent a year by 2019, versus rates below one-digit of traditional solutions.

NetSuite is a good example of a SaaS solution. According to the disclosure of the company, they offer solutions for companies of various segments and sizes and already having customers of certain representation, such as Groupon. "Animated by numbers like these, ERP vendors such as SAP, Oracle and Epicor have accelerated the offering of products that can compete against competitors such as NetSuite" [11]. SAP is already working on an on-demand solution for small and medium-sized businesses called "Business ByDesign," another great example of SaaS. The SaaS model for small businesses is very attractive. The overall advantages of Cloud Computing can be imported into the SaaS ERP model. The possibility of turning capital (capex) expenses into operational (opex) is a big differential for SMEs. The possibility of not having to shift resources and efforts of the company to IT management to deal with servers, routers, software, among others, is also a great differential. It relieves some of the administrative pressure. It is also much faster to have a SaaS deployment. There is no need to buy, install and configure servers and in many cases you can test the application before buying it with agility and ease, unimaginable things for the ERPs marketed today on-premise.

The SaaS ERP model via the Internet browser can also eliminate the need for specific operating systems, allowing companies to install open source alternatives and reduce costs. Other advantages include easier collaborative work between enterprise units and less concern for backups, since keeping data secure and reliable is a cloud responsibility [12]. The main disadvantages are in the relative loss of control over data, the issue of security and personalization. About security, SaaS servers know that this is the main focus to be neutralized and, precisely because of this, they rely on security technologies that many SMEs would not be able to afford alone. As for customization, the SaaS model will gradually allow modules and applications to be added to the central ERP, tending towards a PaaS model simultaneously. In addition, the need for a stable internet connection also presents another challenge for a greater adoption of a SaaS ERP, especially in countries with poor telecommunications infrastructure [11]. Finally, it is also worth noting that in the long term the investment needed to install a traditional ERP can match the consecutive operational costs of a SaaS model.

Saced, Juell-Skielse and Uppstrom of the University of Stockholm published in 2010 a study on the adoption of ERPs in the Cloud Computing model. The analysis will be more focused on the barriers, since they will serve as a basis for criticism. Among the challenges, many companies have already made high investments in traditional ERPs. However, IT typically depreciates very fast and migration to the cloud can be gradual. The lack of large pioneering companies in the use of cloud ERPs is also pointed as a barrier, which is natural but tends to reverse over time. The study also points to international legal barriers, and the issue of server uptime and the Internet connection of companies. These issues have already been discussed earlier, as has the privacy and security issue. However, two barriers are essential to this article: the need for customization and integration and interoperability.

The history of infrastructure technology needs standards. A major limitation for the railroads during their emergence was that "lines are unevenly distributed and critical variables, such as track gauges, coupling mechanisms, and even time zones, still have to be standardized" [6]. This was extremely damaging to the development of railroads. "Infrastructure technologies [6] offer much greater profitability when shared than when used alone" [6].

Producing an ERP is an effort of technology and knowledge, for all that it carries. When a company acquires the right to use an ERP, it also acquires a way to handle processes and run a business. Companies should choose an ERP that fits their processes and not impose processes on it. However, this practice is not usual today. ERPs, when treated as proprietary technology, make the cost of an adaptation outweigh the advantages of differentiation. "Smart companies know that peculiar processes are the key to competitive advantage. However, when it comes to complex applications, the economics of buying a ready-made version has become so attractive that few companies can justify the cost and risk of developing a new system from scratch "[6].

In the paper by Mendes and Escrivao (2002) on the adoption of traditional ERPs (ie, pre-cloud or on-premise ERPs) in SMEs, this question becomes very evident. "Process analysis is a fundamental activity in the adoption of an ERP and is not always performed. It is a time-consuming step that requires professionals with knowledge of the business and the objectives of a company "[13]. And when the analysis is carried out, the costs and difficulty in enforcing differentiation are very great. "The ERP interface with other systems is not easy, since in general, they have proprietary interfaces and adapt them or adapt existing systems to these interfaces requires development effort and programming of tests that can become very costly for the company "[13]. Often, companies do not take these costs into account in the ERP budget, and what happens is an adjustment to what the ERP imposes. In 
short, it presents as a great barrier and difficulty for the SMEs the high cost to customize the acquired ERP.

When we treat ERPs as proprietary technologies, we are subject to these barriers. However, with the cloud one has the possibility of seeing information systems as an infrastructure technology, allowing integration and cross-data, giving companies the possibility to add customized applications, guaranteeing competitive differentiation and, at the same time, maintaining a base of common data. There is a SaaS CRM called SalesForce.com that exemplifies this process. Chris Anderson in the book "The Long Tail" of 2006 presents SalesForce.com as the long tail of software. The company found a way to differentiate itself, beyond simply being a CRM that did not need to be installed and came as a service. "His method of offering software online could also create the conditions for hundreds of smaller developers, many of them in low-cost places like India, to reach the same customers." He realized that SalesForce.com could run an infrastructure, a platform for the niche market, of specific business needs, to be met.

"Autonomous developers could create an application for a specific niche, such as performance appraisal or human resource recruitment, that would run on Salesforce servers, in integration with the company's other software"[14]. Google has a suite of business applications via SaaS - Google Apps for Business - from Email, Calendar, Office Suite, and more.

Recently the company launched the Google Apps Marketplace, a space where you can find applications that integrate with GoogleApps, in the same way as SalesForce. "More than 200 different types of apps were available at Google's Market Place six months after opening in September 2010 and have been used more than 4 million times. [15] The integration between on-demand ERP and a platform approach is promising "[15]. The examples do not stop there. Facebook also allows applications to run on its platform. As you can see, it seems like a trend to have the cloud as an infrastructure, allowing integration and customization. In the previous examples, Google Apps and SalesForce work as a kind of infrastructure / platform for applications.

Finally, to imagine the acquisition of an ERP service under a SaaS platform capable of evolving to allow its customization in a less costly way, possible with the PaaS concept, seems to be an unavoidable way of looking at these advantages alone. But all this evolution given in a future of uncertain time can bring greater consequences than all the advantages now easily enjoyed.

\section{IN ADDITION TO BUSINESS INTEGRATION}

Seeing IT as a "commoditized" infrastructure technology can enable the integration of an organization into an integration between organizations - whether through the supply chain, partner companies, subsidiaries and headquarters, franchising, etc. After all, if companies are running under one platform, integration between their applications can become easier and more robust. It would be possible, for example, to create groups and choose what kind of information to share or not with particular organizations. Pires and Camargo (2010) pondered supply chain integration from the perspective of cloud computing. According to them, increasing customer demand, especially in relation to service quality, has made organizations aware of the importance of properly managing the supply chain to promote a sustainable competitive advantage over their competitors by bringing a collaborative approach with focus in relationships and in partnerships along the chain. But what happens in practice is that "despite the great effort of
Information and Communication Technologies (ICT) for integration and joint management, there are still some difficulties related to the information systems used by companies for this purpose" [16]. According to the authors, "the lack of standardization of systems, the need to use different types of software or the strong dependence on B2B portals means that not all the benefits of efficient supply chain management are achieved" [16].

As we see, cloud computing can provide this standardized environment conducive to such integration, so necessary in today's scenario. "Currently, in the most competitive industrial areas, it is no longer sufficient to only integrate the operations, structures and internal infrastructures with the company's competitive strategy. Organizations that better integrate critical internal processes with suppliers and customers and thus build a supply chain with clear objectives and well-defined procedures tend to obtain better competitive performances "[16].

Despite this need, Pires and Camargo also reinforce that the collaboration between the supply chain has a complicated nature, because it involves the sharing of cultures, business definitions and strategic objectives that do not always go in the same direction. There must be limits on sharing information between organizations, as there is a whole issue involving the privacy and security of a company. However, supply chain integration is not a distant dream. Large automakers operating in Brazil have their integrated supply chain using a real-time satellite communication standard developed by them.

Aalst [17] states that SaaS is particularly interesting because many organizations work with similar processes and that we must be able to configure these processes so that they can differentiate and at the same time support what is common between organizations. He argues that by providing these "configurable services" in the cloud we have the chance to enable cross-organizational process mining. Thus organizations can learn from each other and have a basis for improving their processes.

According to Aalst [17], there may be cases in which companies want to share information. "In this case, crossorganizational process mining is used to perform benchmarking with different organizations and differences in performance are analyzed" [17]. However, he states that this would only be possible in a non-competitive environment, such as organizations belonging to the same holding company, franchises, municipalities, courts, etc. The author presents as an example the CoSeLoG Project (Configurable Services for Local Governments) in implementation in the Netherlands. "As there are 430 municipalities in the Netherlands and all of them promote similar services and run simulated processes, the use of SaaS technology can potentially be very beneficial to local governments. [17] The goal of this project is to create an infrastructure in the cloud for these municipalities "[17]. Aalst reiterates the need for the services to be configurable, since, although similar, the processes vary from municipality to municipalities either by demographic, political or administrative factors. "Cross organizational process mining is not a threat and municipalities want to share information and experiences to learn from each other. Therefore, a cloud infrastructure widely used by municipalities can help establish evidence-based best practices obtained by cross-organizational process mining "[17]. To sum up, if the issue was to integrate departments of the same organization, today, with the cloud, we can think of integrating organizations in a more facilitated way, since the cloud would represent a unified, agile and standardized platform for applications. 


\section{A CLOUd INTELLIGENCE SCENARIO}

What we propose in this topic is to take this approach to the extreme by analyzing an inevitable consequence if tomorrow happens what is expected today. That is, a common IT business infrastructure, which, therefore, will be data unification allowing comparison between organizations. One can allow more configurability, autonomy interoperability. If a SaaS / PaaS infrastructure were so customizable, adaptable, interoperable, modifiable, robust, secure, and all the other qualities that cloud computing can offer, we can assume that many companies would be interested in having the service.

Some time ago, in order to have a good email provider with reasonable storage capacity, it was necessary to subscribe to some special service with providers. Over the years, the price of storage space on the web has become increasingly cheaper and companies have managed to free up more space for their users for free, under the use of advertising or small fees. Let's take a look, for example, that Google Mail offers almost eight gigabytes to its free subscribers, and the company says that number will continue to rise. In addition to having good capacity, webmail is of quality, has uptime of almost $100 \%$, good integration with offline and online services and is relatively customizable. It would be possible to say that few Gmail users would exchange service for one paid because it is very difficult to reach competitive differentials that exceed Google's email cloud. Nevertheless, it was this same company that "founded" the term cloud computing. In 2006, Eric Schmidt - CEO of the company - used the term to describe how Google handled its data centers.

We will then make an analogy between various SaaS available on the Internet that are very successful with the public and have or support a powerful business infrastructure in the cloud. More recent estimates estimate that nearly $13 \%$ of the world's population has an account on Facebook's "social network." Even more curious is that Facebook - as well as several Google tools, for example - does not charge for its services to the general public. What would be possible if $13 \%$ of the world's companies were using common information systems, with the same service provider, through cloud computing?

What we can assume is that as long as it is possible to reduce some of the negative points of using the cloud (such as feeling insecure about lack of privacy), we can have a mass adoption of many companies, and if this happens, of a new world for business life. Moving further, we assume that the system is free. Let's see that in the old model of ERP, they are not as customizable, expensive, costly, imposing processes, and yet companies are forced into those sacrifices to get what they want (or what they most want ). The service offered would be everything the companies want and need and at no cost. It may seem very absurd, after all, how can a company function without charging for its services? However, on the Internet we have several examples of services offered free to users.

When the price of gigabytes is worth cents, we can afford that as long as we have another product coupled to the platform in the cloud. And this product exists, if it is called intelligence.

Facebook plans to raise $\$ 5$ billion in the NYSE and what makes it worth having is simple: data and information. "It's what you put, write, play, share, read, comment on, enjoy, and nudge that made the company make \$ 1 billion in 2011. That may now make it the seventh most valuable technology company in the world [18]. Through a mix of advertising and intelligence, Facebook gets its billing. "Intelligent systems analyze data streams generated by user actions and create 'profile baskets' for anyone who wants to exploit them. [18]
Over time, they become real oceans of data in which companies can fish what consumers want to know "[18].

Duncan J. Watts in the book "Everything is Obvious" (2011) proposes that for many experiments in the field of social sciences, economics and politics has a great challenge mainly as regards the size and scale necessary to these experiments and social networks basis for this to occur. According to him, "online searches, online media and e-commerce are increasingly drawing conclusions about the intentions and actions of people around the world" [19]. The author stresses the privacy-related issue, but states that "these technologies also have enormous scientific potential, enabling us for the first time in history to observe, with great fidelity, the real-time behavior of large groups and even societies as a all "[19].

On Facebook there are already applications for research purposes - or obtaining data for such. Watts introduces an application for the social network called FriendSense, whose goal was to study homophilia - a principle that goes against the argument that opposites attract - a question that generates much controversy among scholars. If it had not been for the power of the Facebook cloud, the experiment would not have occurred in the real world, as it would be very costly and would take years. In the cloud, it took weeks. According to the author, "the increasing availability of observational data, coupled with the ability to conduct experiments on a previously unimaginable scale, has allowed social scientists to imagine a world where at least some forms of collective human behavior can be measured and understood "[19].

Facebook could do more, and that's what makes the company so valuable. But having information on $13 \%$ of the world's population is above all an ethical challenge, even when "anonymizing" the data. Nicholas Carr in "The Great Shift" [19] cites a New York Times report by Michael Barbaro and Tom Zeller Jr. In 2006, AOL released a survey report of 657,000 subscribers. The company judged that the information would be useful to researchers, but sought to anonymize the profiles in the form of numbers. The reporters then decided to find out who the user was "4417749" just by what he was researching. Within hours the user was recognized because her search records said a lot about her. The web is a spider's web, the question is whether it's worth being a prey. Many users now "accept" the lack of privacy in exchange for the benefits and ease that many Internet services offer. If this happens to people, it can occur with organizations, that is, accept the lack of privacy in exchange for the best business information system service that exists. Recently Google was accused of manipulating Web browsers to track users' browsing [20]. It is not known how society will deal with privacy "breaches", but its concept seems to be gradually changing.

The same cloud ERP platform shared by companies using certain standards will enable a very interesting crossreferencing of data and information. As systems tend to have data from the entire departmental extension of a company, we may have in hand a possibility of data mining that can anticipate trends and bring new concepts to business management, especially in the case of benchmarking. In short, if there ever was a "Facebook of the companies", it would be possible to know the companies in the same way that is currently sought to know the consumers. Enterprise cloud computing was what the giants of the new millennium needed to take another step toward gaining information: from people to business.

One can think of, for example, financial analyzes of companies almost in real time, searching for inter- and intrasectoral cause and effect correlations, performance and productivity indicators, among others. That is, we can be 
talking about the evolution of BI and data mining to a sort of artificial intelligence of business management.

We can cite three questions that usually a CEO wants to have the answer: "where do we need to invest?"; "How are we in relation to the competition?"; "How do we mitigate the risk?". Let's see that in relation to the first question, if the CEO had in hand an analysis of the summary of the investments of other companies in his sector he would know how to respond. And that's perfectly conceivable in our cloud-intelligence model. The second question can also be answered and almost real-time, when one knows how the average cash flow of the companies in the sector is, for example, the average invested $\mathrm{R}$ \& D of the competition, the number of new contracts effected, over, employee satisfaction, among others. The third question requires a little more $\mathrm{BI}$ (Business Intelligence) and data mining for your answer. But interesting data can be extracted from the cloud infrastructure. It is interesting to note that in order to answer these questions one need not necessarily identify the companies. A grouping of industry data would suffice. With the model we could move forward in the search for answers to questions such as the return on investments in advertising and marketing, investment in training and training and what the real return on investment in IT for companies.

Uncertainty could be reduced, risks would be mitigated. If, at any point in time, an IT company can achieve this scenario with statistical significance, it can be said that it will have the power of business predictability in hand and will be able to sell or make available the information, commercial profile packages. It would be like driving a car with the aid of a GPS, indicating the market movements, industry indicators and the shortest way to achieve the objectives. Driving in unfamiliar environments would not be more problematic. Strategic business management would have profound transformations. It is true that many financial data of public companies are available for public consultation. However, the financial dimension of a company is not the only one that has impact on their future, we must analyze, for example, intellectual capacity, processes, development. And such data, for example, can be found in the database of an ERP in the cloud.

\section{CONCLUSION}

The purpose of this paper was to explore two of the main possibilities created by a mass adoption of Information Systems supported by the cloud infrastructure: the greater integration between companies (evolution of intra-enterprise integration already attainable today) and the leverage of decision-making processes based on the real-time analysis of large amounts of data. Such adoption must still be better studied, especially with regard to its unfolding and its barriers, such as the lack of privacy and copyright, and the very infrastructure provided by the cloud does not have fully mature concepts. Another point considered as a source of further studies refers to the presence of few players who have the infrastructure of the cloud, visible in the current market, and its possible consequences. The analysis of data resulting from the adoption of systems based on cloud computing, for example, would undergo changes arising from this domain.

\section{REFERENCES}

[1] R. Buyya, C.S. Yeo, S. Venugopal, J. Broberg, I. Brandic, Cloud computing and emerging IT platforms: Vision, hype, and reality for delivering computing as the 5th utility, Future Gener. Comput. Syst. 25 (6) (2009) 599616.

[2] N. Grozev, R. Buyya, Inter-cloud architectures and application brokering: taxonomy and survey, Softw. Pract. Exp. 44 (3) (2014) 369-390.

[3] Y. Mansouri, A.N. Toosi, R. Buyya, Data storage management in cloud environments: Taxonomy, survey, and future directions, ACM Comput. Surv. (2017) 1-51.

[4] D. Petcu, G. Macariu, S. Panica, C. Crciun, Portable cloud applications: From theory to practice, Future Gener. Comput. Syst. 29 (6) (2013) 1417-1430.

[5] Z. Wu, H.V. Madhyastha, Understanding the latency benefits of multi-cloud webservice deployments, SIGCOMM Comput. Commun. Rev. 43 (2) (2013) 13-20.

[6] D. Bernstein, E. Ludvigson, K. Sankar, S. Diamond, M. Morrow, Blueprint for the intercloud - protocols and formats for cloud computing interoperability, in: Proceedings of the 4th International Conference on Internet and Web Applications and Services, 2009, pp. 328-336.

[7] K. Zhang, X. Zhou, Y. Chen, X. Wang, Y. Ruan, Sedic: Privacy-aware data intensive computing on hybrid clouds, in: Proceedings of the 18th ACM Conference on Computer and Communications Security, 2011, pp. 515526.

[8] X. Xu, X. Zhao, A framework for privacy-aware computing on hybrid clouds with mixed-sensitivity Data, in: 17th IEEE International Conference on High Performance Computing and Communications, 7th IEEE International Symposium on Cyberspace Safety and Security, and 12th IEEE International Conference on Embedded Software and Systems, 2015, pp. 1344-1349.

[9] A.N. Toosi, R.O. Sinnott, R. Buyya, Resource provisioning for data-intensive applications with deadline constraints on hybrid clouds using aneka, Future Gener. Comput. Syst. (2017). http://dx.doi.org/10.1016/j.future.2017.05.

[10] B. Rochwerger, C. Vzquez, D. Breitgand, D. Hadas, M. Villari, P. Massonet, E. Levy, A. Galis, I.M. Llorente, R.S. Montero, Y. Wolfsthal, K. Nagin, L. Larsson, F. Galn, An architecture for federated cloud computing, in: Cloud Computing, John Wiley \& Sons, Inc., 2011, pp. 391-411.

[11] R. Buyya, R. Ranjan, R.N. Calheiros, InterCloud: Utilityoriented federation of cloud computing environments for scaling of application services, in: Proceedings of the 10th International Conference on Algorithms and Architectures for Parallel Processing, 2010, pp. 13-31.

[12] B. Varghese, N. Wang, S. Barbhuiya, P. Kilpatrick, D.S. Nikolopoulos, Challenges and opportunities in edge computing, in: IEEE International Conference on Smart Cloud, 2016, pp. 20-26.

[13] F.P. Tso, D.R. White, S. Jouet, J. Singer, D.P. Pezaros, The glasgow raspberry Pi cloud: A scale model for cloud computing infrastructures, in: 33rd IEEE International Conference on Distributed Computing Systems Workshops, 2013, pp. 108-112.

[14] A. Sathiaseelan, A. Lertsinsrubtavee, A. Jagan, P. Baskaran, J. Crowcroft, Cloudrone: Micro clouds in the sky, in: Proceedings of the 2nd Workshop on Micro Aerial Vehicle Networks, Systems, and Applications for Civilian Use, 2016, pp. 41-44.

[15] Y.S.S.A. Elkhatib, B.F. Porter, H.B. Ribeiro, M.F. Zhani, J. Qadir, E. Rivire, On using micro-clouds to deliver the fog, IEEE Internet Comput. 21 (2) (2016) 8-15.

[16] M. Villari, M. Fazio, S. Dustdar, O. Rana, R. Ranjan, Osmotic computing: A new paradigm for edge/cloud integration, IEEE Cloud Comput. 3 (6) (2016) 76-83. 
[17] N. Wang, B. Varghese, M. Matthaiou, D.S. Nikolopoulos, ENORM: A framework for edge node resource management, IEEE Trans. Serv. Comput. (ISSN: 19391374) PP (2017). http://dx.doi.org/10.1109/TSC.2017.2753775.

[18] K. Gai, M. Qiu, H. Zhao, L. Tao, Z. Zong, Dynamic energy-aware cloudlet-based mobile cloud computing model for green computing, J. Netw. Comput. Appl. 59 (C) (2016) 46-54.

[19] M. Satyanarayanan, P. Bahl, R. Caceres, N. Davies, The case for VM-based cloudlets in mobile computing, IEEE Pervasive Comput. 8 (4) (2009) 14-23.

[20] G.A. McGilvary, A. Barker, M. Atkinson, Ad hoc cloud computing, in: Proceedings of the IEEE 8th International Conference on Cloud Computing, 2015, pp. 1063-1068. 